\title{
Actitudes hacia las ciencias de los estudiantes de NM1 del Colegio San Sebastián de Los Andes de la red de colegios educaUC: un enfoque pedagógico- social a la enseñanza de las ciencias
}

\author{
Israel Romero Rivas, educaUC, Chile
}

\begin{abstract}
Resumen: El presente estudio tiene como objetivo identificar las actitudes hacia las ciencias de los estudiantes de NMI mediante la aplicación del Test of Science Related Attitudes (TOSRA), el cual estandariza mediante subescalas las actitudes relacionadas con la ciencia, tales como implicaciones sociales de la ciencia, estilos de vida de los científicos, entre otros. Con la aplicación durante un semestre de un taller de ciencias, basado en la experimentación de temas asociados al currículum actual, los estudiantes expresan un cambio en sus actitudes. Para validar estos cambios, se aplicará un Post-test del TOSRA, el cual puede ser analizado estadísticamente aplicando McNemar y la prueba T, comparando simultáneamente las actitudes según el sexo de la muestra.
\end{abstract}

Palabras clave: actitudes hacia la ciencia, TOSRA

Abstract: The present study aims to identify the attitudes toward Science Students NM1 through the Application of Science Related Attitudes Test (TOSRA), which standardizes by subscales the attitudes related to science, such as social implications of science and lifestyles of scientists, among others. With the implementation of a science workshop during one semester based on the experimentation of the current curriculum's associated topics, the students show a change in their attitudes. In order to validate these changes, a TOSRA posttest will be taken. This test can be analyzed statistically by applying McNemar and the T test, comparing the attitudes simultaneously according to the sex of the sample.

Keywords: Attitudes toward Science, TOSRA

\section{Objetivos}

Tentificar y comparar las actitudes hacia las ciencias de los estudiantes de NM1 mediante la apli1 cación del test de TOSRA.

Comparar según sexo las actitudes hacia las ciencias de los estudiantes de NM1.

Insertar en el plan de estudio un programa de ciencias experimental para determinar un cambio en las actitudes hacia las ciencias de los estudiantes de NM1.

\section{Pregunta de investigación}

¿Cómo influye la incorporación de un taller experimental en las actitudes hacia las ciencias de los estudiantes de NM1 del colegio San Sebastián de Los Andes?

\section{Hipótesis}

H1: Si se incorpora en el plan de estudio de NM1 un taller de ciencias, entonces los estudiantes mejorarán las actitudes hacia las ciencias.

H0: Si se incorpora en el plan de estudio de NM1 un taller de ciencias, no se observarán cambios en las actitudes hacia las ciencias.

H2: Si se incorpora en el plan de estudio de NM1 un taller de ciencias, entonces existirán diferencias en las actitudes hacia las ciencias en hombres y mujeres.

Revista Internacional de Aprendizaje en Ciencia, Matemáticas y Tecnología

Volumen 1, Número 1, 2014, <http://sobrelaeducacion.com>, ISSN 2386-8791

(C) Global Knowledge Academics. Israel Romero. Todos los derechos 
H0: Al incorporar en el plan de estudio de NM1 un taller de ciencias, no existirán diferencias en las actitudes hacia las ciencias en hombres y mujeres.

\section{Justificación del problema}

La selección de este nivel se basa en la desmotivación existente al estudio del taller de ciencias, el cual fue modificado curricularmente en el 2014, con el fin de obtener logros significativos en el aprendizaje y mayor apreciación hacia el estudio de las ciencias.

Además, como la educación en Chile es generalizada durante los ocho primeros años de escolaridad, cuando alcanzan el noveno año (primero medio), las ciencias naturales se dividen en Química, Física y Biología, donde los estudiantes no visualizan la integración de estas tres áreas en la revisión curricular de sus contenidos y acciones prácticas.

\section{Marco teórico}

En Chile, la enseñanza de las ciencias ha tenido distintos cambios curriculares que han generado un descontento social y una desmotivación por parte de los estudiantes para su estudio.

En el Marco Curricular de la Educación para el sector de ciencias se plantea que a futuro la marginalidad estará ligada a la carencia de conocimientos básicos en ciencias y a las formas de pensamiento propias a la investigación científica. Es por ello que resulta significativo estudiar las actitudes hacia la ciencia de los estudiantes, debido a las repercusiones que tiene el aprendizaje de las disciplinas científicas en las decisiones que toman los estudiantes en el futuro y en la percepción de los alcances de la ciencia y la tecnología en materias globales.

Según Allport (1935), una actitud corresponde al "estado de preparación" o disposición mental o psicológica y neutral, que se organiza a través de la experiencia y que influye en la respuesta del sujeto. Las actitudes hacia la ciencia están relacionadas con el componente afectivo de cada individuo, haciendo referencia a la valoración de las personas hacia diversos objetos actitudinales, tales como, la ciencia escolar, los científicos, la preferencia por una carrera afín con ciencia, implicancias sociales asociadas con las ciencias, entre otros.

$\mathrm{Al}$ analizar las actitudes hacia la ciencia, investigaciones exponen la influencia de ciertas variables, como lo es el sexo de los estudiantes. En Chile, los resultados de la prueba PISA 2006 se observaron diferencias entre hombres y mujeres en el aprendizaje en ciencias, existiendo una diferencia significativa a favor de los hombres (448 puntos versus 426 puntos mujeres) manteniéndose en el año 2009. Estas diferencias en los desempeños en el área de las ciencias tienen consecuencias en la motivación para aprender la disciplina y en la actitud hacia el aprendizaje de ella en la escuela, lo que finalmente afecta los caminos que seguirán los estudiantes en el ámbito educacional y profesional.

\section{Metodología}

\section{Muestra}

La muestra constó de 65 alumnos (32 mujeres y 33 hombres) de primer año medio del Colegio San Sebastián de Los Andes perteneciente a la red educaUC. La edad promedio de los estudiantes es de 14 años con un mínimo de 13 años y un máximo de 16 años. Los estudiantes pertenecen a dos cursos del nivel de estudio (Primero medio A y Primero medio B). Después de la aplicación del pretest de TOSRA, se trabajará en el taller de ciencias dos horas semanales en el laboratorio de ciencias, según horario de trabajo de cada curso, hasta fines del primer semestre del año en curso, para luego evaluar las actitudes con la aplicación del post test del TOSRA. 


\section{Instrumentos}

\section{Escala de actitudes hacia la ciencia}

Se utilizó el Test of Science Related Attitudes (TOSRA, "Escala de actitudes relacionadas con la ciencia"). Esta escala, orientada a estudiantes de educación secundaria, está compuesta por 70 ítems, distribuidos en las siguientes subescalas: Implicaciones sociales de la ciencia (S); Estilo de vida de los científicos (N); Actitud hacia la investigación científica (I); Adopción de actitudes científicas (A); Agrado por las clases de ciencia (E); Interés por la ciencia durante el tiempo libre (L); Interés en carreras científicas (C).

Cada subescala se compone de 10 afirmaciones frente a las cuales los estudiantes deben señalar su agrado de acuerdo o desacuerdo en una escala de tipo Likert de cinco puntos. Las subescalas de TOSRA presentan una confiabilidad que oscila entre 0,67 y 0,93, y la confiabilidad global es de 0,90. La aplicación del pretest fue el 18 de marzo de 2014 y el post-test el 18 de junio de 2014 con una duración de 45 minutos, aproximadamente.

\section{Taller de ciencias}

El taller de ciencias permite aumentar el interés de los estudiantes en el área de las ciencias, el conocimiento de conceptos y procedimientos científicos, y la adquisición de nuevas competencias. Por consiguiente, las experimentaciones deben ser entendidas como herramientas metodológicas que permiten mejorar el aprendizaje científico escolar.

Desde esta perspectiva, el programa que se incorporará dispone de una selección e incorporación de actividades de experimentación que permiten promover la expresión de las propias ideas de los estudiantes sobre un determinado fenómeno y el planteamiento de preguntas significativas que faciliten el aprendizaje y la construcción del conocimiento científico, acompañadas con sus planificaciones por semana de trabajo pedagógico. El taller se desarrolla semanalmente (dos horas pedagógicas) en el laboratorio de ciencias, según horario de los cursos en estudio.

\section{Análisis de datos}

Se realizó un pretest del TOSRA con el fin de determinar inicialmente las actitudes de los estudiantes frente a las ciencias. Inicialmente, los análisis estadísticos son de tipo descriptivo e inferencial. Se utilizó la Prueba t para analizar la comparación de las actitudes de los estudiantes según sexo con un nivel de significancia del $p<0,05$. Finalmente, se aplicó el post test del TOSRA para evaluar los cambios de actitud hacia las ciencias de los estudiantes.

\section{Resultados}

\section{PRE TEST}

Del total de subescalas del test, el que presentó la media más alta frente a una actitud positiva es la Adopción de actitudes científicas (A) (media $=18,54 ; \mathrm{SD}=2,44)$ y la subescala que presentó la media más alta frente a una actitud negativa fue las Implicaciones sociales de la ciencia (S) (media $=17,62 ; \mathrm{SD}=2,68)$. Por consiguiente, la subescala que presentó la media más baja frente a una actitud positiva fue el Interés en carreras científicas $(\mathbf{C})$ (media $=13,00 ; \mathrm{SD}=3,77$ ) y la subescala que presentó la media más baja frente a una actitud negativa fue el Interés por la ciencia en el tiempo libre $(\mathbf{L})($ media $=14,00 ; \mathrm{SD}=3,94)($ Tabla 1$)$. 
REVISTA INTERNACIONAL DE APRENDIZAJE EN CIENCIA, MATEMÁTICAS Y TECNOLOGÍA

Tabla 1: Estadística descriptiva de la muestra

\begin{tabular}{|c|c|c|c|c|c|c|c|}
\hline & Media & Mediana & Moda & SD & Varianza de la muestra & Curtosis & Coeficiente de asimetría \\
\hline S (+) & 17,95 & 18,00 & 18,00 & 2,45 & 5,98 & $-0,20$ & $-0,32$ \\
\hline S (-) & 17,62 & 18,00 & 18,00 & 2,68 & 7,21 & $-0,04$ & $-0,14$ \\
\hline $\mathbf{N}(+)$ & 17,49 & 17,00 & 21,00 & 3,38 & 11,44 & $-0,86$ & $-0,11$ \\
\hline $\mathbf{N}(-)$ & 15,03 & 15,00 & 15,00 & 2,16 & 4,69 & 4,57 & 0,81 \\
\hline $\mathbf{I}(+)$ & 17,66 & 18,00 & 18,00 & 3,37 & 11,38 & $-0,72$ & $-0,18$ \\
\hline $\mathbf{I}(-)$ & 15,35 & 15,00 & 13,00 & 2,68 & 7,20 & $-0,12$ & 0,01 \\
\hline $\mathbf{A ~ ( + )}$ & 18,54 & 19,00 & 20,00 & 2,44 & 5,94 & $-0,20$ & $-0,74$ \\
\hline $\mathbf{A ~ ( - )}$ & 17,51 & 18,00 & 18,00 & 2,67 & 7,13 & 0,80 & $-0,56$ \\
\hline $\mathbf{E ~ ( + )}$ & 14,66 & 15,00 & 14,00 & 3,90 & 15,23 & $-0,67$ & 0,21 \\
\hline $\mathbf{E ~ ( - )}$ & 16,98 & 17,00 & 15,00 & 2,87 & 8,27 & $-0,08$ & 0,14 \\
\hline $\mathbf{L}(+)$ & 13,49 & 13,00 & 13,00 & 4,48 & 20,07 & 0,13 & $-0,30$ \\
\hline $\mathbf{L}(-)$ & 14,00 & 14,00 & 16,00 & 3,94 & 15,50 & $-0,27$ & $-0,03$ \\
\hline $\mathbf{C ~ ( + )}$ & 13,00 & 13,00 & 13,00 & 3,77 & 14,19 & $-0,08$ & 0,27 \\
\hline $\mathbf{C ~ ( - )}$ & 14,55 & 14,00 & 12,00 & 3,28 & 10,75 & $-0,06$ & 0,28 \\
\hline
\end{tabular}

Fuente: Elaboración propia, 2014.

El gráfico 1 ilustra lo señalado anteriormente.

Gráfico 1: Actitudes hacia las ciencias según las medias de pretest

\section{Actitudes hacia las ciencias: Pre-Test}

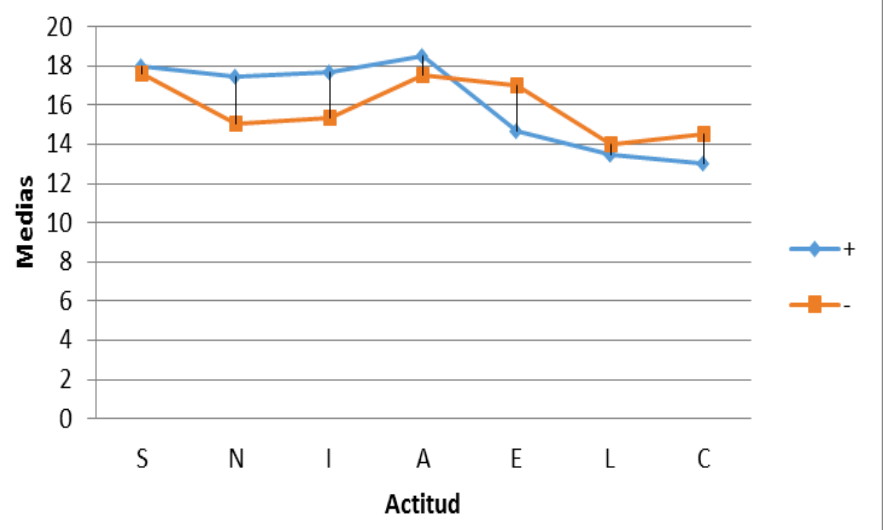

Fuente: Elaboración propia, 2014.

Analizando las diferencias de las medias en función del sexo de la muestra completa, se observa que no hay diferencias significativas en las actitudes frente a las ciencias entre los hombres y las mujeres $(\mathrm{t}=0,34, \mathrm{p}>0,05)$ (tabla 2$)$. 
Tabla 2: Prueba t: Diferencias de las media en función del sexo

\begin{tabular}{|l|r|r|}
\hline & \multicolumn{1}{|c|}{$H$} & \multicolumn{1}{c|}{$M$} \\
\hline Media & 16,10 & 15,85 \\
\hline Varianza & 3,51 & 3,46 \\
\hline Observaciones & 14,00 & 14,00 \\
\hline Varianza agrupada & 3,49 & \\
\hline Diferencia hipotética de las medias & 0,00 & \\
\hline Grados de libertad & 26,00 & \\
\hline Estadístico t & 0,34 & \\
\hline P(T<=t) una cola & 0,37 & \\
\hline Valor crítico de t (una cola) & 1,71 & \\
\hline P(T<=t) dos colas & 0,73 & \\
\hline Valor crítico de t (dos colas) & 2,06 & \\
\hline
\end{tabular}

Fuente: Elaboración propia, 2014.

\section{POST TEST: Prueba de McNemar}

De acuerdo a los resultados del post-test del TOSRA, existen cambios significativos en las subescalas relacionadas con el agrado por las clases de ciencias (E) y el interés por la ciencia en el tiempo libre (L). (Tabla $\mathrm{N}^{\circ} 3$ ).

Tabla 3: Resultados de la significancia, según McNemar

\begin{tabular}{|c|c|c|c|c|c|c|c|c|c|c|c|c|c|c|}
\hline & \multicolumn{2}{|c|}{ S } & \multicolumn{2}{|c|}{ N } & \multicolumn{2}{c|}{ I } & \multicolumn{3}{c|}{ A } & \multicolumn{3}{|c|}{ E } & \multicolumn{3}{|c|}{ L } & \multicolumn{3}{c|}{ C } \\
\hline & + & - & + & - & + & - & + & - & + & - & + & - & + & - \\
\hline P<0.05 & $\mathbf{0 . 7 7}$ & $\mathbf{0 . 7 5}$ & $\mathbf{0 . 7 9}$ & $\mathbf{0 . 0 7}$ & $\mathbf{0 . 2 1}$ & $\mathbf{0 . 8 4}$ & $\mathbf{0 . 3 4}$ & $\mathbf{1}$ & $\mathbf{0 . 0 2}$ & $\mathbf{0 . 0 0 1}$ & $\mathbf{0 . 0 2}$ & $\mathbf{0 . 3 9}$ & $\mathbf{0 . 6 3}$ & $\mathbf{0 . 3 1}$ \\
\hline Sig. & Nsig & Nsig & Nsig & Nsig & Nsig & Nsig & Nsig & Nsig & S & S & S & Nsig & Nsig & Nsig \\
\hline
\end{tabular}

Fuente: Elaboración propia, 2014.

El gráfico 2 ilustra la tabla anterior.

Gráfico 2: Resultados de la significancia de cada actitud, según McNemar

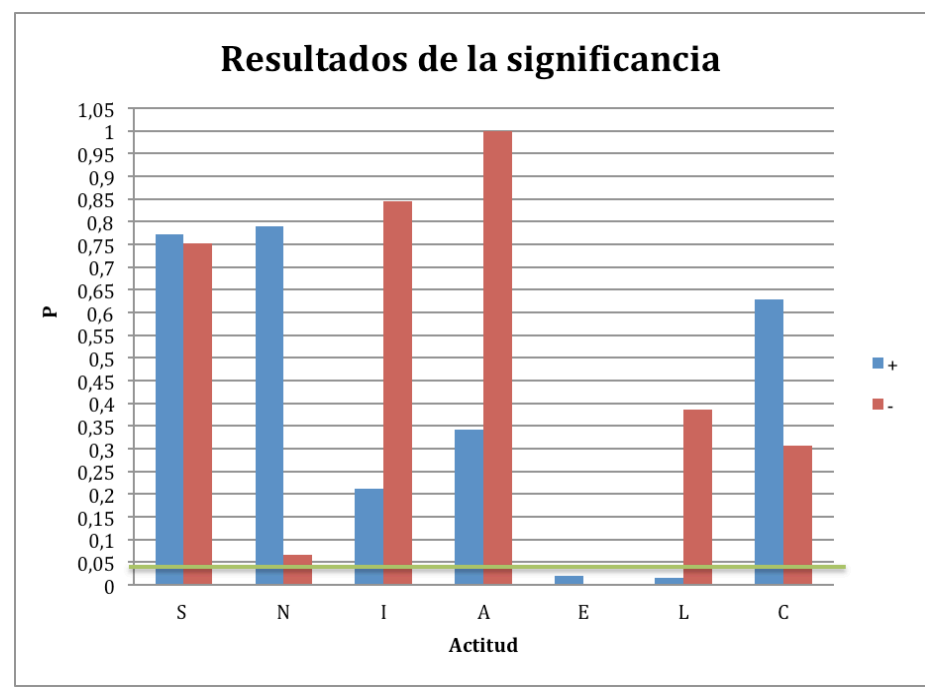

Fuente: Elaboración propia, 2014.

Analizando las diferencias de las medias en función del sexo de la muestra completa, se observa que no hay diferencias significativas en las actitudes frente a las ciencias entre los hombres y las mujeres $(\mathrm{t}=0,93, \mathrm{p}>0,05)$ (Tabla 4$)$. 
Tabla 4: Prueba t: Diferencias de las medias en función del sexo

\begin{tabular}{|l|l|l|}
\hline & \multicolumn{1}{|c|}{$M$} & \multicolumn{1}{c|}{$H$} \\
\hline Media & 17.1428571 & 16.478355 \\
\hline Varianza & 4.22967462 & 2.91533214 \\
\hline Observaciones & 14 & 14 \\
\hline Varianza agrupada & 3.57250338 & \\
\hline Diferencia hipotética de las medias & 0 & \\
\hline Grados de libertad & 26 & \\
\hline Estadístico t & 0.93016308 & \\
\hline $\mathrm{P}(\mathrm{T}<=\mathrm{t})$ una cola & 0.1804216 & \\
\hline Valor crítico de t (una cola) & 1.70561792 & \\
\hline $\mathrm{P}(\mathrm{T}<=\mathrm{t})$ dos colas & 0.36084321 & \\
\hline Valor crítico de t (dos colas) & 2.05552944 & \\
\hline
\end{tabular}

Fuente: Elaboración propia, 2014.

Gráfico 3: Diferencias de actitud, según género

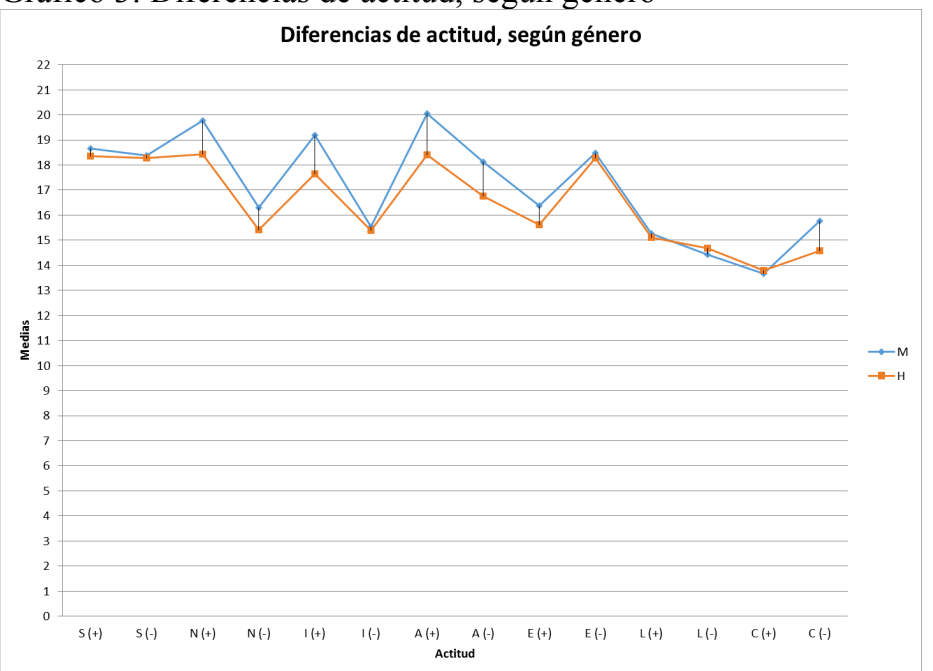

Fuente: Elaboración propia, 2014.

Sin embargo, analizando las diferencias de las medias en función del sexo de cada subescala, se observa que existe una diferencia significativa sólo en la adopción de actitudes científicas en mujeres y no en hombres. (Tabla 5).

Tabla 5: Prueba t: Adopción de actitudes científicas, según género

\begin{tabular}{|l|l|l|}
\hline \multicolumn{1}{|c|}{$\boldsymbol{M}$} & \multicolumn{1}{c|}{$\boldsymbol{H}$} \\
\hline Media & 20.0625 & 18.3939394 \\
\hline Varianza & 5.22177419 & 9.37121212 \\
\hline Observaciones & 32 & 33 \\
\hline Varianza agrupada & 7.3294252 & \\
\hline Diferencia hipotética de las medias & 0 & \\
\hline Grados de libertad & 63 & \\
\hline Estadístico t & 2.48417569 & \\
\hline $\mathrm{P}(\mathrm{T}<=\mathrm{t})$ una cola & 0.00782801 & \\
\hline Valor crítico de t (una cola) & 1.66940222 & \\
\hline $\mathrm{P}(\mathrm{T}<=\mathrm{t})$ dos colas & 0.01565601 & \\
\hline Valor crítico de t (dos colas) & 1.99834054 & \\
\hline
\end{tabular}

Fuente: Elaboración propia, 2014. 


\section{Conclusiones}

De acuerdo a los resultados obtenidos en el pretest, se observó una tendencia en la adquisición de conductas científicas por el grupo en estudio, lo que implica que los estudiantes al ser individuos concretos (según edad), están más dispuestos a plantearse preguntas que les permitan resolver problemas en aspectos contextualizados, procurando la rigurosidad en el trabajo y promoviendo el pensamiento crítico. Es fundamental, el planteamiento de preguntas clase a clase con el fin de mantener la atención de los estudiantes en los aspectos en desarrollo.

A pesar de que no existen diferencias según género de la muestra, posterior a la intervención, la subescala mencionada, manifiesta una diferenciación significativa en mujeres que en los hombres.

Además, en el pretest presentaron una actitud negativa frente a las implicaciones sociales de la ciencia, por lo que dentro del programa sería fundamental analizar las oportunidades y amenazas que las ciencias tienen en la sociedad actual y no sólo abordar las temáticas de manera distante con los estudiantes.

Otra situación importante a inferir corresponde al interés por la ciencia durante el tiempo libre, la cual expresa un cambio significativo de forma positiva en la actitud de los estudiantes. Esto refleja que la intervención pedagógica realizada por el docente titular del módulo, permite que los estudiantes cuestionen en cada instante y espontáneamente los distintos eventos del mundo científico. Esto va ligado a los logros de aprendizaje que el profesor propone en su proceso metodológico, junto a los indicadores de evaluación.

Cabe señalar que la subescala agrado por las clases de ciencia manifiesta una condición muy particular, ya que tanto las actitudes positivas como negativas presentan cambios significativos, siendo esta última la que más enmarca este resultado. Con respecto a esta subescala, se puede inferir que el módulo de ciencias al estar estructurado con el método tradicional de la experimentación, genera profundamente mayor rechazo al módulo, de modo que es fundamental modificar el programa con un diseño de actividades donde el estudiante pueda crear y compartir las vivencias experimentales (indagación). Desde un plano psicológico, en esta edad los estudiantes buscan la autonomía de sus acciones, respondiendo a que los alumnos en este nivel son demandantes de interaccionar de forma directa con los eventos estudiados.

De acuerdo a la prueba estadística t, no existen diferencias significativas entre hombres y mujeres frente a las actitudes hacia las ciencias.

Finalmente, estudiar las actitudes hacia las ciencias de los estudiantes, permite conocer las repercusiones que tiene el aprendizaje de las disciplinas científicas en las decisiones que toman estos en su futuro y en la percepción de los alcances de la ciencia y la tecnología en materias globales. 


\section{REFERENCIAS}

Acevedo, J. (2007). Consensos sobre la naturaleza de la ciencia: fundamentos de una investigación empírica. Revista Eureka sobre Enseñanza y Divulgación de las Ciencias, 4(1), pp. 42-66.

Allport, G.W. (1935). Attitudes. In C. Murchison (ed.), Handbook of social psychology (pp. 798844). Worcester, MA: Clark Univ. Press.

Escudero, T. (1995). La evaluación de las actitudes científicas. Didáctica de las ciencias experimentales, 2(4), pp. 33-41.

Fraser, B. (1981). Test of science related attitudes. Melbourne: Australian Council for Educational Research.

Gardner, P. (1975). Attitudes to science: A review. Studies in Science Education, 2, pp. 1-41.

Manassero, M. y Vásquez, A. (2001). Instrumentos y métodos para la evaluación de las actitudes relacionadas con la ciencia, la tecnología y la sociedad. Enseñanza de las ciencias, 20(1), pp. 15-27.

Navarro, M. y Forster, C. (2012). Nivel de alfabetización científica y actitudes hacia la ciencia en estudiantes de secundaria: comparaciones por sexo y nivel socioeconómico. Pensamiento Educativo. Revista de Investigación Educacional Latinoamericana, 49(1), pp. 1-17. Recuperado de http://pensamientoeducativo.uc.cl/index.php/pel/article/view/507/1082

OCDE (2006). PISA 2006. Marco de la evaluación. Conocimientos y habilidades en ciencias, matemáticas y lectura. Recuperado de www.oecd.org/dataoecd/59/39732471.pdf.

Vásquez et al. (2006). Actitudes del alumnado sobre ciencia, tecnología y sociedad evaluadas con un modelo de respuesta múltiple. Revista electrónica de investigación educativa, 8(2), pp. 1-37. Recuperado de http://redie.uabc.mx/vol8no2/contenido-vasquez2.html

\section{SOBRE EL AUTOR}

Israel Romero Rivas: Es profesor de Química y Biología. Trabajó activamente durante ocho años en colegios y educación superior. Actualmente, se desempeña como coordinador de ciencias de la red de colegios educaUC. Durante su formación profesional y ejercicio docente no ha dejado de investigar en educación, en la línea de estrategias de enseñanza y actitudes hacia las ciencias. Su compromiso en educaUC es entregar una formación de calidad que requiere de esfuerzos en todos los ámbitos y de todos los actores del proceso educativo basados en tres pilares fundamentales: formación en valores, gestión de calidad y excelencia académica. Cada uno de ellos cuenta con acciones y programas orientados a alcanzar las metas, favoreciendo con un trabajo bien hecho, a la red y sus integrantes. El trabajo enviado, que se encuentra en proceso de investigación, al ser presentado ante la comisión de especialistas de esta convocatoria, demostrará que a pesar de que sus alumnos tienen una mirada lejana hacia las ciencias por el alto nivel de complejidad, tienen la convicción de que en su revisión experimental, adoptarán actitudes científicas que les permitirán sociabilizar con sus pares, discutiendo y contribuyendo acerca de sus innovaciones y aportes al mundo actual. 